\title{
The p35 relative, p49, inhibits mammalian and Drosophila caspases including DRONC and protects against apoptosis
}

\author{
AM Jabbour ${ }^{1,2,3}$, PG Ekert ${ }^{4,5}$, EJ Coulson ${ }^{5}$, MJ Knight ${ }^{1,2}$, \\ DM Ashley ${ }^{1,2,3}$ and CJ Hawkins ${ }^{\star 1,2,3}$ \\ ${ }^{1}$ Department of Haematology and Oncology, Royal Children's Hospital, Parkville \\ 3052, Australia \\ 2 Murdoch Children's Research Institute, Royal Children's Hospital, Parkville \\ 3052, Australia \\ ${ }^{3}$ Department of Paediatrics, The University of Melbourne, Parkville 3010 , \\ Australia \\ ${ }^{4}$ Department of Neonatology, Royal Children's Hospital, Parkville 3052, \\ Australia \\ 5 The Walter and Eliza Hall Institute, The Royal Melbourne Hospital, Parkville \\ 3050, Australia \\ * Corresponding author: CJ Hawkins, Department of Haematology and \\ Oncology, Royal Children's Hospital, Parkville 3052, Australia \\ Tel: (613) 9345 5823; Fax: (613) 9345 6524; \\ E-mail:hawkinsc@cryptic.rch.unimelb.edu.au
}

Received 4.4.02; revised 7.8.02; accepted 20.8.02

Edited by $\mathrm{G}$ Salvesen

\begin{abstract}
This study characterized the ability of a new member of the $\mathrm{p} 35$ family, p49, to inhibit a number of mammalian and insect caspases. p49 blocked apoptosis triggered by treatment with Fas ligand (FasL), Tumor necrosis factor-related apoptosisinducing ligand (TRAIL) or ultraviolet (UV) radiation but provided negligible protection against apoptosis induced by the chemotherapeutic drug cisplatin. The caspase cleavage site in p49 was determined, and mutation of the $\mathrm{P} 1$ residue of this site abolished the ability of p49 to inhibit caspases, implying that p49 inhibits caspases through an analogous suicide-substrate mechanism to p35. Unlike p35, p49 inhibited the upstream insect caspase DRONC.

Cell Death and Differentiation (2002) 9, 1311-1320. doi:10.1038/ sj.cdd. 4401135
\end{abstract}

Keywords: apoptosis; baculovirus; p35; p49; caspase

Abbreviations: UV, ultraviolet; NPV, nucleopolyhedrovirus; $A c$, Autographa californica; Spli, Spodoptera littoralis; Bm, Bombyx mori; Splt, Spodoptera litura; Ha, Heliothis armigera; TRAIL, tumor necrosis factor-related apoptosis-inducing ligand; PCR, polymerase chain reaction; GFP, green fluorescent protein; IAP, inhibitor of apoptosis; FasL, Fas ligand; CDDP, cisplatin; TE, Tris $\mathrm{HCl} 10 \mathrm{mM}$ pH8, EDTA $1 \mathrm{mM}$; AFC, 7-Amino-4-(trifluoromethyl)coumarin; FBS, fetal bovine serum

\section{Introduction}

Apoptosis is controlled through evolutionarily conserved molecular pathways that signal activation of a family of downstream effector proteases known as caspases. Apoptosis fulfills a number of vital roles in metazoans, including elimination of virally infected cells (reviewed $\mathrm{in}^{1}$ ). A number of viruses employ strategies to evade this defensive apoptosis, including expression of proteins that inhibit caspases. ${ }^{2}$ Such viral caspase inhibitors fall into three groups based on homology. CrmA is a serine protease inhibitor (serpin) and is a relatively specific inhibitor of Caspases- 1 and $-8 .^{3}$ The inhibitor of apoptosis (IAP) family comprises baculoviral, insect and mammalian members that function, at least in part, by directly binding and inhibiting active caspases. ${ }^{4}$ The third group of caspase inhibitors is the p35 family of inhibitors.

The p35 family of anti-apoptosis proteins constitutes a distinct class of caspase inhibitors for which no cellular homologs have been reported to date. The best studied p35 protein is encoded by the Autographa californica nucleopolyhedrovirus (AcNPV) genome. ${ }^{5,6} \mathrm{~A}$ less potent relative from Bombyx mori nucleopolyhedrovirus (BmNPV) has also been cloned..$^{7,8}$ p35 genes from other baculoviral strains have been identified but not published as yet. AcNPV-p35 strongly protects Caenorhabditis elegans, Drosophila melanogaster and mammalian cells against diverse apoptotic stimuli (reviewed $\mathrm{in}^{9}$ ). This potent apoptosis suppression derives from p35's ability to directly inhibit almost all caspases studied, regardless of species of origin. ${ }^{10,11}$ p35 is also capable of inhibiting gingipain- $R$ (a distant relative of the caspase family). ${ }^{12}$

The mechanism of caspase inhibition by p35 has been reported as suicide-substrate inhibition, in which cleavage of p35 by the caspase ${ }^{13}$ results in a conformational change, causing the covalent attachment of the amino-terminal fragment to the caspase. ${ }^{14-18}$ Interestingly, the activity of the Drosophila apical caspase DRONC is unaffected by p35, ${ }^{19,20}$ as is that of a candidate DRONC homolog from Spodoptera frugiperda, Sf-caspase-X. ${ }^{21}$ p35 has also been reported to be incapable of inhibiting human Caspase- 9 in vivo. $^{22,23}$

A second member of the p35 family, termed p49, shares approximately $50 \%$ amino acid identity with AcNPV-p35. p49 was isolated from Spodoptera littoralis nucleopolyhedrovirus (SpliNPV) by virtue of its ability to rescue insect cells from apoptosis triggered by infection with a p35 defective baculovirus. ${ }^{24}$ In addition, Manji and Friesen refer to unpublished data demonstrating that unlike p35, p49 can block Sf-caspase-X activity. ${ }^{25}$ The sequence of a second p49 gene from Spodoptera litura nucleopolyhedrovirus (SpltNPV) has been deposited in the database (accession AF207549), but has not yet been characterized.

This study characterized the ability of SpliNPV - p49 to inhibit a number of mammalian and insect caspases, and to block apoptotic stimuli. The caspase cleavage site in p49 was determined, and mutation of the $\mathrm{P} 1$ residue of this site 
abolished the ability of p49 to inhibit caspases, implying that p49 inhibits caspases through an analogous suicidesubstrate mechanism to p35. Unlike p35, p49 inhibited the upstream insect caspase DRONC.

\section{Results}

\section{Analysis of sequence homology within the p35 family of proteins}

The p35 family consists of members encoded by genomes of different baculoviral strains. Sequence comparisons between members of the p35 family were performed to identify regions of potential functional importance (Figure 1). This comparison of predicted amino acid sequences encoded by the four p35 genes and two p49 genes identified to date deviated slightly from that published previously, in which only one p49 and one p35 were compared. ${ }^{24}$ In accordance with the report of Du et al., caspase cleavage of p49 was predicted to occur after residues TVTD. ${ }^{24}$ Strong sequence homology between all members of the p35 family was evident in the amino terminal half of the proteins, and at their carboxyl termini. The two p49 proteins contained insertions of short stretches of amino acids in the carboxyl half of the proteins, compared to their p35 relatives. Previous mutagenesis studies of AcNPV-p35 indicated that insertions of di-peptides into these regions, predicted to form intervening loops between $\alpha$-helices and $\beta$ sheets, ${ }^{14}$ did not alter the activity of p35.
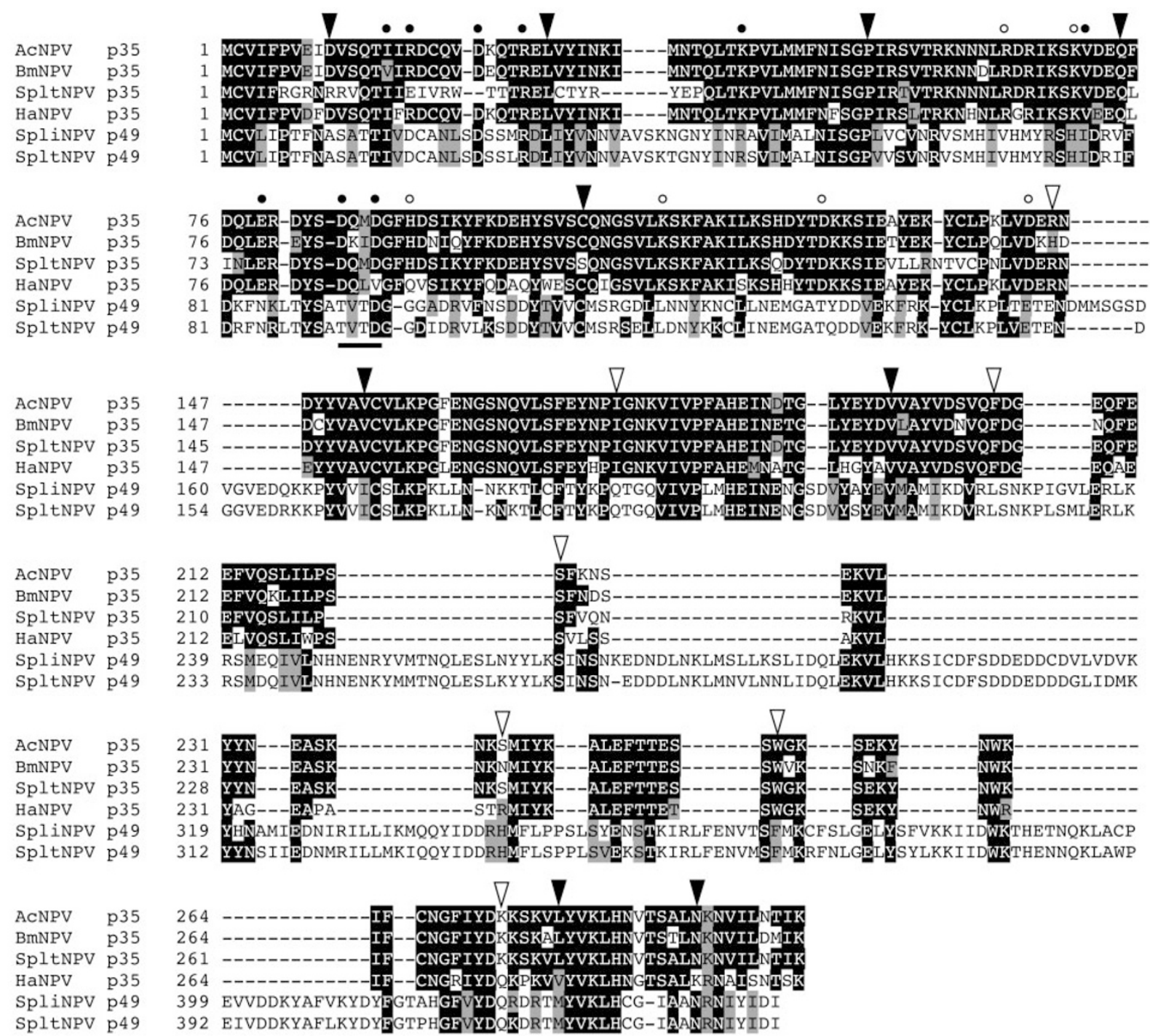

Figure 1 Multiple alignment of known p35 and p49 proteins. Black shading represents residues which are identical in at least $50 \%$ of sequences, gray shading indicates residues with similarity to conserved residues. The caspase cleavage site of AcNPV-p35 (underlined) aligns with caspase recognition sites within the homologs indicating conserved function. Mutations within AcNPV-p35 have been marked according to functional outcomes. ${ }^{13-18}$ Black circles are above amino acids where change results in loss of function. Open circles represent single amino acids where change does not affect function. Black triangles indicate where a di-amino acid insertion inhibits function whereas open triangles indicate where di-amino acid insertions have no effect. Note that residues for which mutations affect function tend to be those which are highly conserved between both the p49 and p35 members, whereas mutations in regions less conserved between family members are often functionally ineffectual. This study focuses on the p49 of Spodoptera littoralis nucleopolyhedrovirus (SpliNPV-p49) 


\section{Ability of p49 to inhibit caspase-dependent death in S. cerevisiae}

Many caspases expressed at sufficient levels (or in engineered, auto-activating forms) cause death in the yeast $S$. cerevisiae. When a caspase is co-expressed with an inhibitor, such as p35, survival and growth of the yeast indicates caspase inhibition. ${ }^{26}$ In this study we aimed to test whether, like p35, p49 could inhibit caspases in this system. We generated a series of expression constructs which directed expression of carboxyl terminally FLAG epitope tagged p35 (p35F) and p49 (p49F) at a range of levels. Under control of identical promoters, p49F was expressed at much lower levels than p35F. By expressing p35F under the control of a crippled GAL1/10 promoter (see Materials and Methods) and p49F from the intact GAL1/10 promoter, this difference was lessened, with p35F being expressed at approximately fivefold higher levels than p49F (Figure 2A). These proteins and their untagged counterparts were assayed for caspase inhibitory potential using an autoactivating version of Caspase-3. As shown in Figure 2B, like p35, p49 could inhibit Caspase-3 induced death in yeast. The ability of the tagged proteins to inhibit Caspase-3 (Figure 2B) and other caspases (data not shown) was similar to that of the untagged proteins.

Expression of active forms of human Caspases-2, -3, -4, $-5,-7,-8$, and Drosophila caspases DRONC, drICE and DCP-1 induced death of $S$. cerevisiae (Figure 3 ). To compare the caspase specificity of $p 49$ to that of $p 35$, these proteins or IAPs were expressed in yeast bearing inducible expression plasmids for various lethal caspases.

Like p35F, p49F exhibited broad caspase-inhibitory capability (Figure 3). p35F and p49F protected against Caspases-2, $-3,-7$, drICE and DCP-1 to approximately the same degree. Interestingly, there were some variations in the antagonistic potential of p35F and p49F. p35F inhibited

A
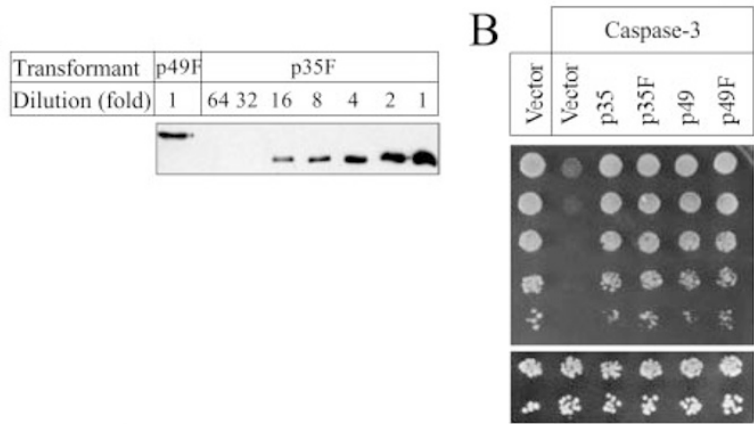

Figure 2 Inhibition by tagged and untagged p49 of Caspase-3 induced death in $S$. cerevisiae. (A) Immunoblotting was used to compare expression levels of FLAG tagged p35 (p35F) and p49 (p49F). Lysate from an induced p49F transformant culture and twofold serial dilutions of a lysate generated from an equivalent amount of an induced p35F transformant culture were subjected to anti-FLAG immunoblotting. (B) Suspensions of yeast co-expressing tagged or untagged p35 or p49 with Caspase-3 were spotted in serial dilutions from top to bottom onto inducing medium (upper panels) and repressing medium (lower panels), to assay protection from caspase- 3 induced lethality. Addition of a carboxyl terminal FLAG-epitope tag did not substantially affect inhibitory potential

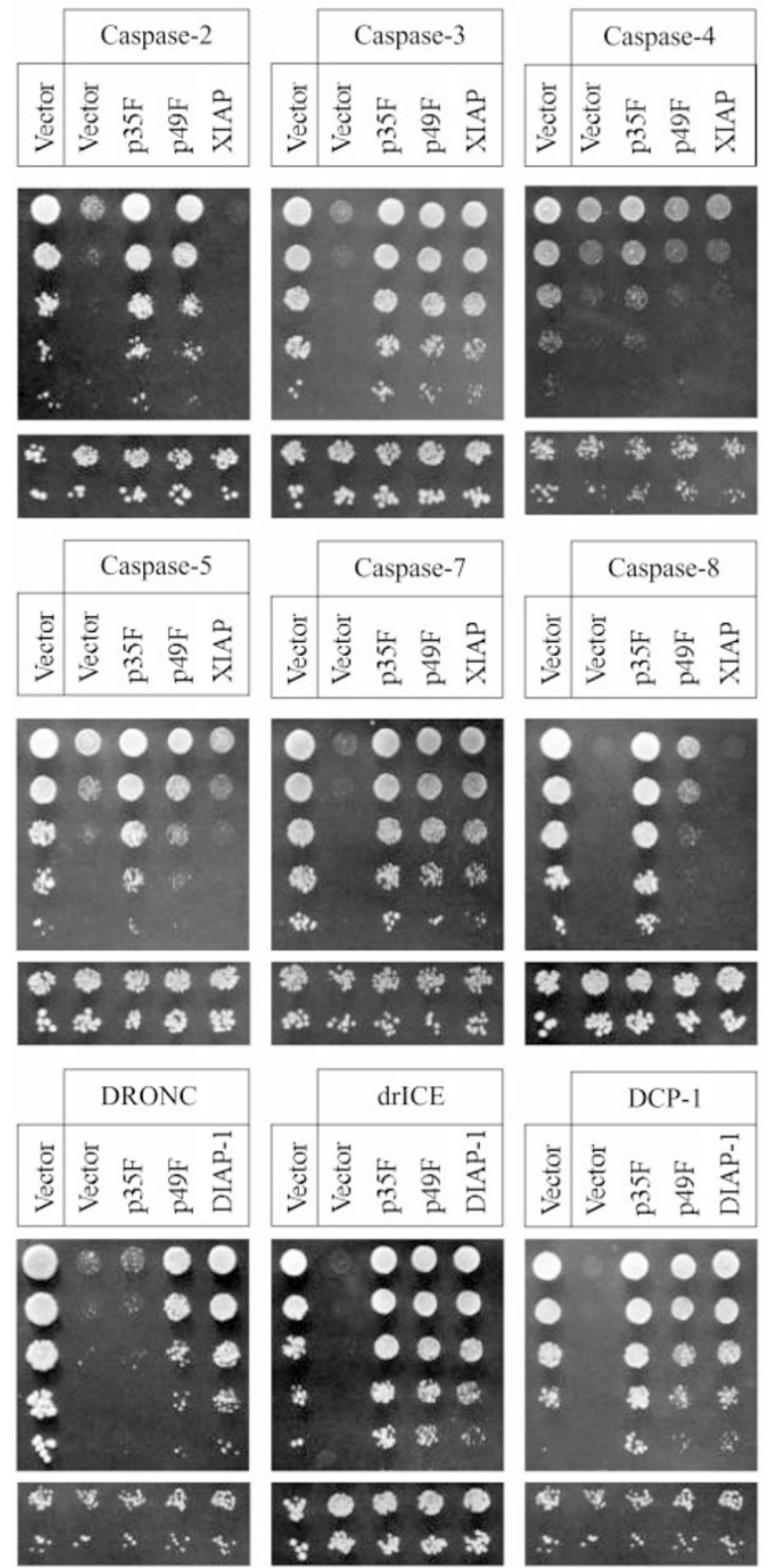

Figure 3 Inhibition of caspase induced death in S. cerevisiae. Expression of autoactivating forms of the indicated caspases (as detailed in Materials and Methods) was enforced in yeast, resulting in lethality when spotted in serial dilutions onto inducing medium (upper panels) but not repressing medium (lower panels). Spotting of co-transformants expressing p35F, p49F, XIAP or DIAP-1 permits visualization of the protection offered by these proteins against the death induced by each caspase. p49F inhibited human Caspases-2, $-3,-5$, -7 , and -8 and Drosophila caspases DCP-1, drICE and DRONC

Caspases -5 and -8 more efficiently than did p49F. Whereas p35F provided partial protection against Caspase-4-induced death, p49F was inactive. We and others have previously demonstrated that DRONC is insensitive to inhibition by p35. ${ }^{19,20}$ In contrast, p49F protection against DRONCinduced death was observed in the yeast system (Figure 3). IAPs can inhibit a subset of caspases, so XIAP and DIAP-1 were used as controls in these assays. As previously demonstrated, XIAP was able to protect against 
Caspases-3, and $-7^{27}$ and DIAP-1 was able to protect yeast from death mediated by drICE, DCP-1 and DRONC. ${ }^{19,26}$

p35 has been reported to be incapable of inhibiting Caspase- 9 in cellular contexts. ${ }^{22,23}$ It would therefore have been useful to determine the ability of p49 to inhibit Caspase-9. Unfortunately active Caspase-9 does not kill yeast, presumably due to differential specificity for vital yeast substrates. ${ }^{28}$ When artificially activated, Caspase- 9 processes pro-Caspase- 3 and yeast death is observed. ${ }^{28}$ As we have shown that both p35 and p49 inhibit Caspase3 , this method would not be feasible to assess the ability of p49 to inhibit Caspase- 9 activity. Hence the ability of p49 to inhibit Caspase-9 could not be determined in this system.

\section{Site directed mutagenesis of the P1 site causes loss of p49 activity}

Previous studies have indicated that the caspases cleave p35 following the amino acid sequence DQMD. ${ }^{87}(11,13)$. Mutation of the DQMD ${ }^{87} \mathrm{P} 1$ aspartate residue to alanine significantly reduced the ability of $\mathrm{p} 35 \mathrm{~F}$ to inhibit Caspases-2 and -3 (Figure 4A,B). Site directed mutagenesis was used to change the predicted $\mathrm{P} 1$ residue of $\mathrm{p} 49$ (D94) to an alanine residue as for $\mathrm{p} 35$. Analysis of $\mathrm{p} 49 \mathrm{~F}^{\mathrm{D} 94 \mathrm{~A}}$ activity when co-expressed in yeast with Caspases-2 or -3 indicated that the protective ability of p49F was completely abolished by the engineered mutation (Figure 4A,B).

To confirm that caspases cleaved p49 at TVTD ${ }^{94},{ }^{35} \mathrm{~S}$ labeled in vitro translated p49 and p49 ${ }^{\mathrm{D} 94 \mathrm{~A}}$ were incubated with recombinant caspases. Cleavage of p35 or p49 correlated with the disappearance of full-length proteins with increasing caspase concentrations. For p49, appearance of a cleavage product was also observed (Figure $4 C, D)$. In contrast, even high concentrations of Caspases-2 and -3 were unable to cleave $\mathrm{p} 49^{\mathrm{D} 94 \mathrm{~A}}$ or $\mathrm{p} 35^{\mathrm{D} 87 \mathrm{~A}}$.

\section{Ability of p49 to inhibit caspase enzymatic activity in S. cerevisiae}

To check that the inhibition of yeast death conferred by coexpression of p49F (Figure 3) was due to inhibition of the enzymatic activity of the caspases, we tested the ability of lysates from various co-transformants to cleave fluorogenic caspase substrates. Caspases-3, -8 and drICE efficiently cleave their preferred synthetic tetrapeptide substrates conjugated to 7-Amino-4-(trifluoromethyl)coumarin (AFC), so were analyzed for susceptibility to p49 inhibition fluorometrically. Consistent with the observation that p35F and p49F inhibited Caspase-3 and drICE-dependent yeast death, lysates co-expressing either p35F or p49F along with active Caspase-3 or drICE cleaved the substrate Ac-DEVD-AFC at a slower rate than lysates expressing the caspases alone (Figure 5A,B). Co-expression of p35F decreased the LEHDase activity of lysates co-expressing Caspase-8, but co-expression of p49F had no significant inhibitory effect (Figure 5C). Lysates from yeast co-expressing a caspase, together with proposed cleavage site mutants of p35F or $\mathrm{p} 49 \mathrm{~F}$, cleaved the substrate at an equivalent rate to lysates from yeast expressing the caspase alone, confirming that
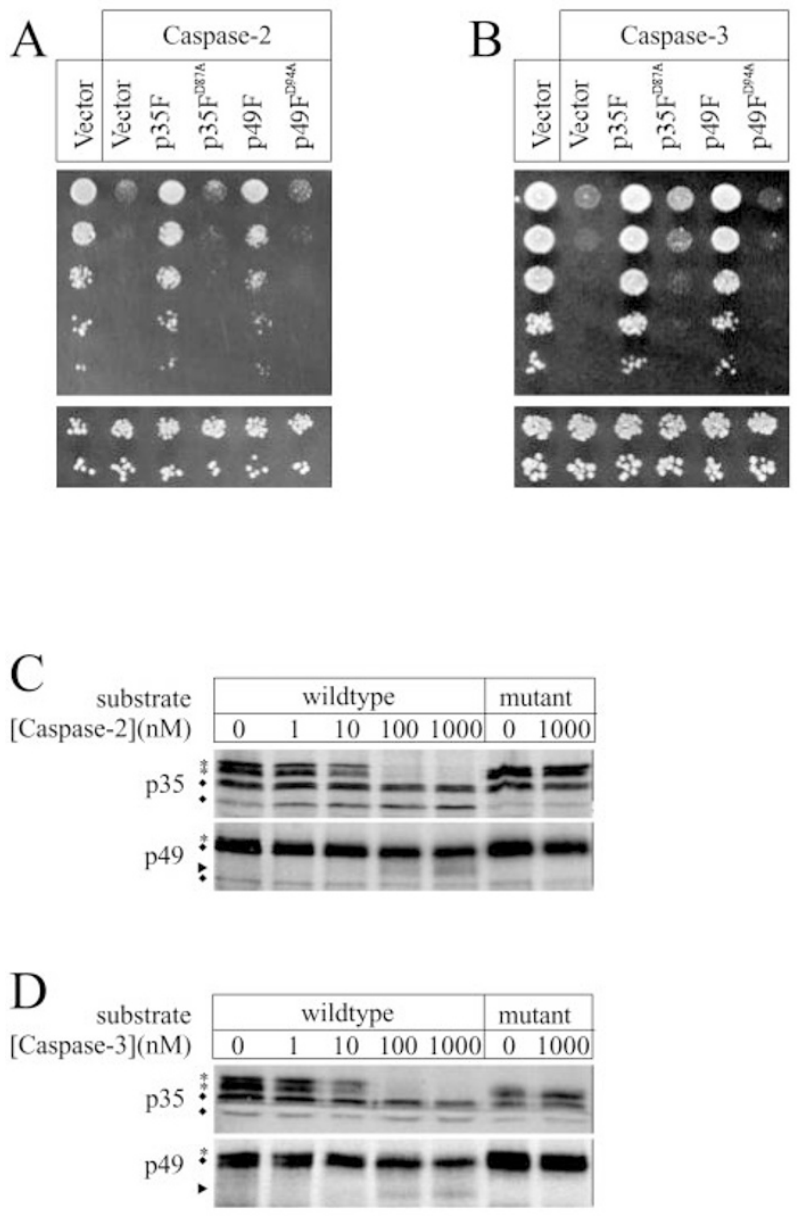

* intact protein $\cdot$ non-specific bands $\boldsymbol{*}$ cleavage product

Figure 4 Determination of caspase cleavage site of $\mathrm{p} 49 . \mathrm{p} 35^{\mathrm{D} 87 \mathrm{~A}}$ and $p 49^{\mathrm{D} 94 \mathrm{~A}}$ mutants were expressed in yeast $(\mathbf{A}, \mathbf{B})$ or generated by radiolabeled in vitro transcription/translation (C, D). (A, B) In contrast to the ability of the wild-type p35 and p49 proteins to inhibit caspase-induced yeast death, the mutants offered no protection. (C, D) When incubated with increasing concentrations of active recombinant Caspase-2 or -3 the wild type proteins were cleaved however the mutant proteins remained unaffected

mutation of the proposed cleavage sites of p35 and p49 abolished function.

\section{The anti-apoptotic ability of p49}

To characterize the ability of p49 to inhibit apoptosis in mammalian cells, plasmids encoding either p35, p49, XIAP or empty vector were co-transfected into three sensitive cell lines together with a marker expression plasmid either directing expression of Green Fluorescent Protein (GFP) or $\beta$ galactosidase. The co-transfected cells were scored for apoptosis either by morphological criteria ( $\beta$-galactosidase co-transfections) or by annexin- $\mathrm{V}$ binding as measured flow cytometrically (GFP co-transfections).

D270 glioblastoma cells are sensitive to treatment with either FasL (Apo1L/CD95L), TRAIL (Apo2L) or cisplatin, 
A

B
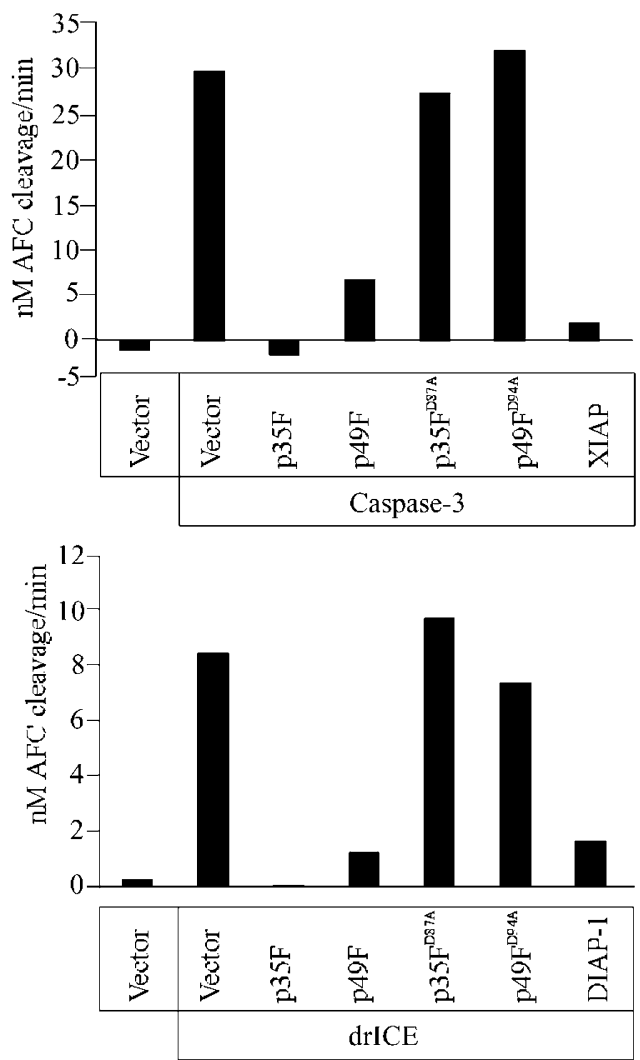

$\mathrm{C}$

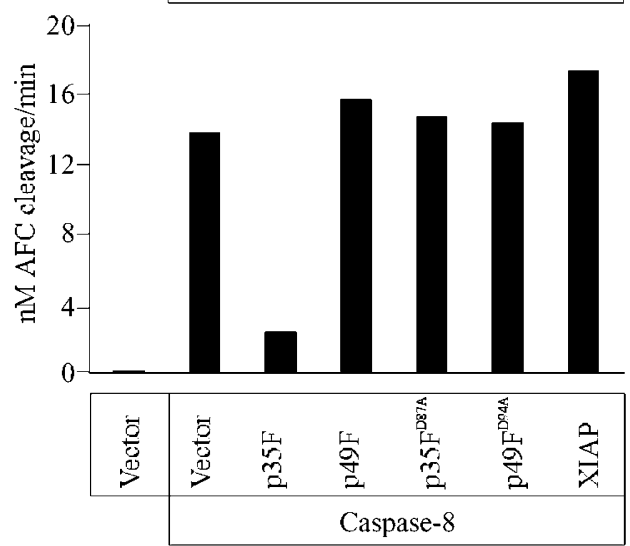

Figure 5 Inhibition of caspase enzymatic activity by p35 and p49. Lysates made from yeast expressing the indicated proteins were incubated with the caspase substrates Ac-DEVD-AFC or Ac-LEHD-AFC. The caspase activity in the lysates was monitored fluorimetrically. The background fluorescence was subtracted and the rate of release of free AFC was calculated as a measure of caspase activity. Lysates from yeast bearing active Caspase-3 (A), drICE (B) and Caspase-8 (C) cleaved the caspase substrates, resulting release of free fluorescent AFC, compared to lysates from control empty vector-transformed yeast. Co-expression of wild-type p35F or p49F (but not their cleavage site mutants) with Caspase-3 (A) or drICE (B) resulted in slower substrate cleavage by those lysates, indicating that p35F and p49F did inhibit the enzymatic activity of the caspases. Co-expression of wild type but not mutant p35F with Caspase-8 (C) yielded less substrate cleavage, whereas the effect of co-expression of p49F on Caspase-8 activity was minimal

whereas D645 glioblastoma cells are susceptible to FasL and TRAIL induced death, but not cisplatin. ${ }^{29}$ D270 cells require mitochondrial amplification for death receptor signaling, whereas D645 cells do not. ${ }^{29}$ NT2 cells are sensitive to apoptosis induced by exposure to UV light. ${ }^{30}$ For all cells and stimuli tested, p49 conferred weaker protection against the apoptotic stimuli than p35 (Figure 6). The degree to which p49 expression rescued the cells from the apoptotic stimuli varied with the treatment and the cell line used. The most striking protection conferred by $\mathrm{p} 49$ was against FasL and TRAIL-induced death in the D270 cell line (Figure 6A,C,G).

\section{Discussion}

Inhibition of caspases has evolved in some viruses, presumably as a mechanism to inhibit defensive apoptosis of host cells which limits viral replication. The high level of evolutionary conservation of cell death pathways has meant that such viral proteins (including p35) are able to function in many systems and have contributed substantially to the elucidation of the role of caspases in many cell death pathways in mammals, worms and insects. The identification of a second member of the p35 family, p49, enhances our understanding of this family of proteins. p49 may, like p35, also be used as a tool to dissect cell death pathways and determine their components. This study represents the first analysis of the caspase inhibitory capabilities of p49 and its capacity to inhibit mammalian cell death.

\section{Inhibition of caspase-dependent yeast death by p49}

We previously developed a system in which yeast can be rescued from caspase-mediated toxicity by caspase inhibitors. This technique was used to evaluate the inhibitory potential of $\mathrm{p} 49$ with respect to a number of caspases.

As expected from its homology to p35, p49 was found to be a broad spectrum caspase inhibitor. p49 could inhibit yeast death mediated by human Caspases-2, -3, -5, -7, -8 and the Drosophila caspases DCP-1, drICE and DRONC. Although in general the protection conferred by p35 and p49 was similar, several differences were notable. The inhibition by $\mathrm{p} 49$ of Caspases- 5 and -8 was weaker than that provided by p35, and p49 could not inhibit Caspase- 4 . In contrast, p49 inhibited the p35 insensitive Drosophila caspase, DRONC. This caspase is somewhat distinct in that it has a non-consensus active site pentapeptide $(P F C R G)^{31}$ and can process substrates with either an aspartate or a glutamate in the $\mathrm{P} 1$ position. ${ }^{19}$ In addition, DRONC was the first caspase shown to be insensitive to inhibition by p35. ${ }^{19,20}$ p35 has also been reported by others to be incapable of inhibiting the processing of Caspase-3 triggered by Cytochrome $c$ treatment of extracts using a reconstituted cell-free system. ${ }^{22}$ Presumably Caspase-3 processing in this system is dependent on Caspase-9, implying that Caspase-9, like DRONC, is insensitive to p35 inhibition. Further evidence that Caspase- 9 is not subject to inhibition by p35 under physiological conditions has also recently been published. ${ }^{23}$ As described in the results section, an assessment of the ability of p49 to inhibit Caspase- 9 was not feasible. 
A

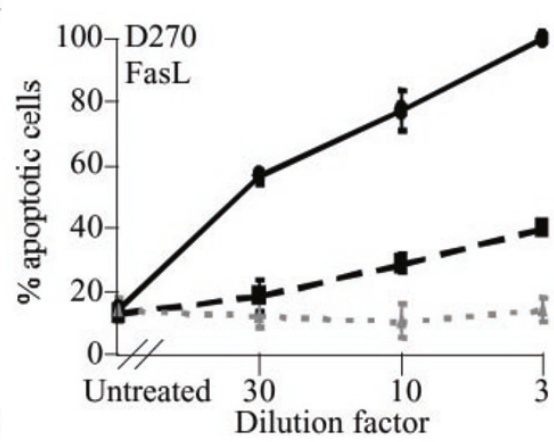

$\mathrm{C}$

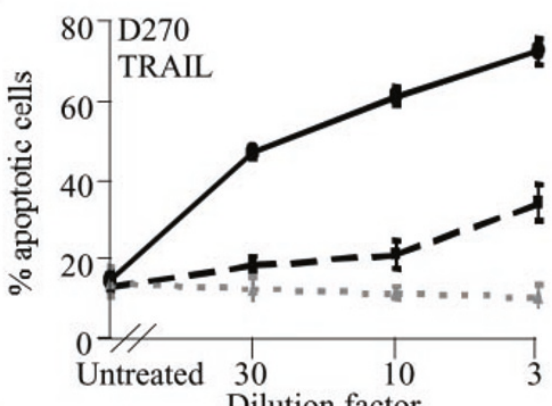

$\mathrm{E}$

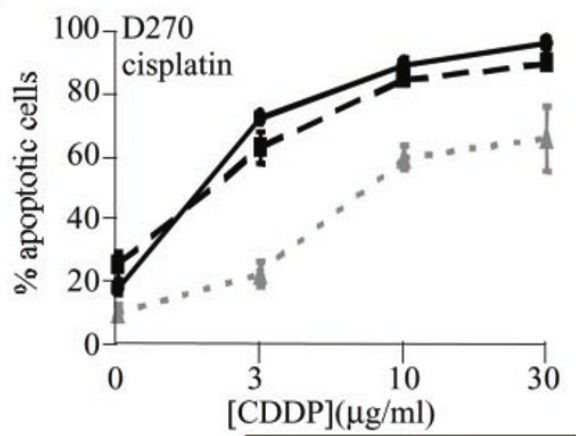

G

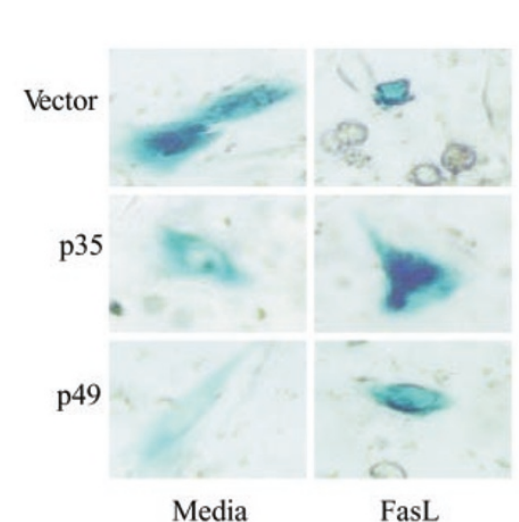

$\mathrm{F}$

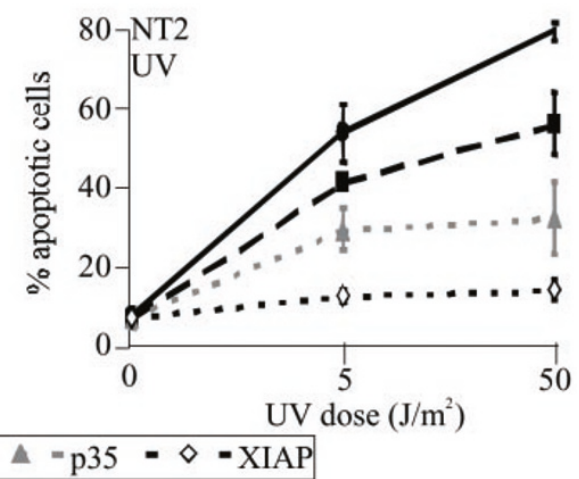

B
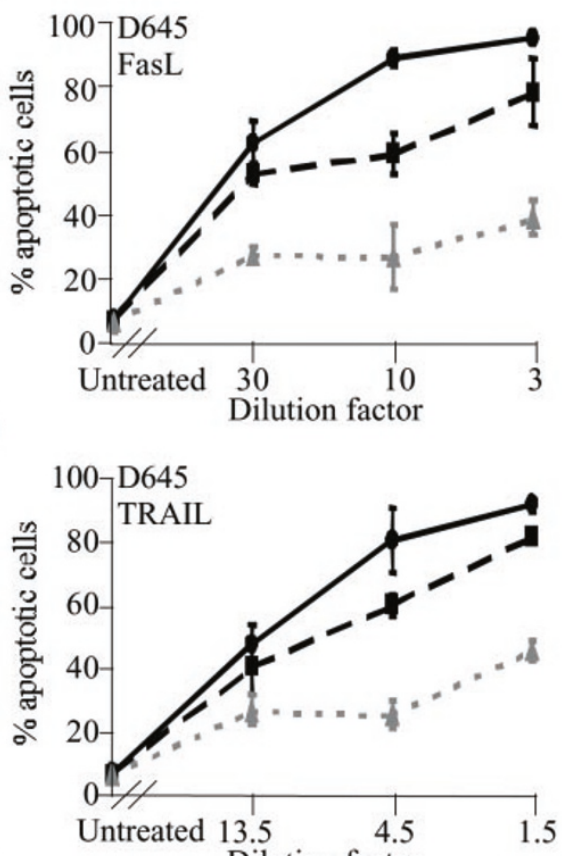

Dilution factor

$\mathrm{H}$

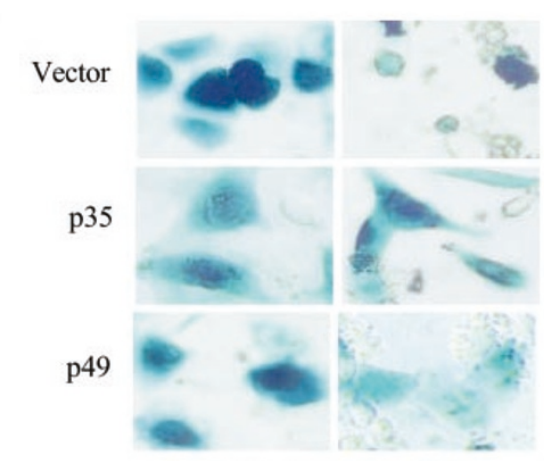

Media

FasL

Figure 6 Inhibition of intrinsic and extrinsic apoptosis stimuli by p49. D270 (A, C, E, G), D645 (B, D, H) or NT2 (F) cells were co-transfected with a marker expression plasmid and either empty vector or p35, p49 or XIAP expression plasmid as indicated. After treatment with the indicated dose of FasL (A, B), TRAIL (C, D), cisplatin $(\mathbf{E})$ or UV radiation $(\mathbf{F})$, the co-transfected cells were scored either microscopically $(\mathbf{A}-\mathbf{E})$ or by flow cytometry $(\mathbf{F})$ for apoptosis. Representative photographs of FasL treated and untreated $\beta$-galatosidase co-transfected D270 (G) and D645 (H) cells are shown 


\section{Structure/function comparison between p35 and p49}

Alignment of the predicted amino acid sequences of the p35 family members identified to date highlighted the regions of homology between the p49 and p35 members of this family, in particular at the amino and carboxyl terminal ends. Stretches of AcNPV-p35, mainly in the carboxyl terminal half, have been previously shown to form loops between $\alpha$-helices and $\beta$-sheets. ${ }^{15-18}$ Mutagenesis of such regions has little effect on p35 function, ${ }^{13,18}$ indicating the absence of a vital role for these regions. The corresponding sections of the two p49 proteins analyzed contained insertions relative to the p35 proteins, consistent with $\mathrm{p} 49$ having a similar structure to $\mathrm{p} 35$. Residues of p35 found to be crucial for function were conserved in both p49 proteins. $^{13,18}$ The predicted reactive site loop of $\mathrm{p} 49$, based on sequence comparison with $\mathrm{p} 35$, encompassed the potential caspase cleavage site TVTD. ${ }^{94}$ As for p35, mutation of this predicted pseudosubstrate site in p49 abolished its caspase inhibitory activity, as discussed below. An unexpected byproduct of the alignment analysis was the demonstration that the predicted pseudosubstrate site of Heliothis armigera nucleopolyhedrovirus (HaNPV)-p35 comprises residues DQLV, lacking a P1 aspartate or glutamate residue. This p35 would therefore be predicted to be an ineffective caspase inhibitor.

\section{Inhibition of caspase enzymatic activity by $\mathbf{p 4 9}$}

Low expression and solubility of p49 using three bacterial expression systems ( $p E T$, pMAL and pGEX) made purification of p49 extremely difficult. Although p49 could be expressed at functional and detectable levels in yeast, it was not possible to isolate and purify p49 from yeast with sufficient yield and purity for quantitative biochemical analyses. Caspases-3, -8 and drICE exhibit sufficiently high rates of cleavage of their preferred tetrapeptide substrates that lysates from yeast expressing these active caspases had detectable fluorogenic substrate-cleaving activity. Lysates from yeast co-expressing p35, p49 or XIAP/DIAP-1 together with Caspase-3 or drICE had dramatically decreased substrate-cleaving ability compared with lysates from yeast expressing each caspase alone, however co-expression of the cleavage site p35 or p49 mutants had no such effect. This confirmed that the protection afforded by p35, p49 and XIAP/ DIAP-1 against drICE or Caspase-3-mediated yeast death was due to inhibition of the enzymatic activity of these caspases and that mutation of the proposed caspase cleavage site of p35 and p49 abolished their caspase inhibitory activity. Consistent with the weak protection conferred by $\mathrm{p} 49$ against Caspase-8 dependent yeast death, cleavage of AC-LEHD-AFC by lysates from yeast expressing Caspase-8 was substantially diminished through co-expression of $\mathrm{p} 35$, but not $\mathrm{p} 49$.

\section{Apoptosis inhibition by $\mathrm{p} 49$}

p35 inhibits a wide range of cell death stimuli in diverse organisms. ${ }^{10,32-42}$ We have shown that like p35, p49 could also inhibit apoptosis induced by UV radiation and by death receptor signaling. The protection conferred by p49 expression was weaker than that conferred by $p 35$. Both proteins exhibited the strongest apoptosis suppression in D270 cells treated with the death ligands. It is interesting that XIAP protected NT2 cells from UV-induced apoptosis better than did p35 or p49, given that the inhibitory potential of XIAP against the caspases tested was less than that of either p35 or p49. The most likely explanation relates to the ability of XIAP to inhibit Caspase-9. XIAP could prevent the Caspase-9/ Caspase-3 amplification loop triggered by UV treatment through inhibiting both caspases. As p35 appears not to inhibit Caspase-9 in cellular contexts, ${ }^{22,23}$ its ability to prevent UV-induced apoptosis would depend entirely on its efficient inhibition of downstream caspases such as Caspase-3 and it is therefore not surprising that the protection offered by p35 is somewhat weaker than XIAP.

As p35 has until recently been considered a universal caspase inhibitor, the inability of p35 to inhibit some forms of apoptosis is one line of evidence to suggest some apoptotic cell deaths may be 'caspase independent'. ${ }^{43}$ Another possible explanation is that such apoptotic pathways may utilize caspases such as DRONC, which are resistant to inhibition by p35. The demonstration that p49 is capable of inhibiting a p35-insensitive caspase may permit its use as a tool to determine potential components of such 'caspase independent' apoptotic pathways.

\section{Materials and Methods}

\section{Sequence comparisons}

Sequences for the known p35 and p49 proteins were aligned using ClustalW v1.7 and adjusted manually to reflect functional homology data. Genbank accession numbers for the sequences used were BmNPV-p35, AY048772; AcNPV-p35, M16821; SpltNPV-p35, Y10254; HaNPV-p35, AF063105; SpliNPV-p49, AJ006751; SpltNPVp49, AF207549.

\section{Plasmid construction}

For yeast experiments, coding DNA sequences were expressed by inducible Gal1/10 promoters. $^{44}$ Plasmids expressing Caspase-3LacZ, Caspase- $7^{53}$, reverse drICE, DCP-1, DIAP-1, and XIAP have been previously described. ${ }^{19,26,28}$ Other plasmids described below were generated by ligating coding sequences into $\mathrm{BamHI} / \mathrm{Xbal}$ digested vectors unless otherwise stated. Caspase-2 was generated by PCR (oligonucleotides 1 and 2), cut with Bg/ll and Xbal and ligated into BamHI/Xbal cut pGALL - (LEU2) and pGALL - (URA). Caspase-4 was generated by PCR (oligonucleotides 3 and 4 ) and ligated into pGALL-(LEU2) and pGALL-(URA). Caspase- 5 (oligonucleotides 5 and 6) was amplified and ligated into pGALL-(LEU2). Caspase- $7^{53}$ was excised from pGALL-(LEU2)-Casp7 $7^{53}$ (Reference ${ }^{26}$ ) and inserted into pGALL-(URA). Caspase-8 (oligonucleotides 7 and 8) was inserted into pGALL-(LEU2). DRONC was cloned from GALL$\left(\right.$ LEU2) ${ }^{19}$ into GALS - (LEU2) and GALL-(URA). PCR was used to clone p49 (oligonucleotides 9 and 10) from pBSK p49 ${ }^{23}$ (kindly supplied by Dr. Nor Chejanovsky). The resulting p49 PCR product was digested and ligated into pGALL-(HIS3). p49 was also amplified (oligonucleotides 11 and 10) and ligated into $\mathrm{pEF}^{45}$ for mammalian expression. The CMV-lacZ plasmid and pEF-p35 have been previously described. ${ }^{45}$ Site directed mutagenesis to generate 
p35 $5^{\mathrm{D} 87 \mathrm{~A}}$ and $\mathrm{p} 49^{\mathrm{D} 94 \mathrm{~A}}$ was performed using PCR. To generate p35 ${ }^{\mathrm{D} 87 \mathrm{~A}}$, the amino terminal and carboxyl terminal fragments were amplified (oligonucleotides 12 and 13, 14 and 15 respectively), digested with $\mathrm{BamHI} / \mathrm{Xhol}$ and $\mathrm{Xhol} / \mathrm{Xbal}$ respectively and cloned into pGALL-(HIS3) and pBluescript SKII+ for in vitro transcription/ translation. To generate $\mathrm{p} 49^{\mathrm{D} 94 \mathrm{~A}}$, the amino terminal and carboxyl terminal fragments were amplified (oligonucleotides 16 and 17, 18 and 19 respectively), digested with BamHI/Sacll and Sacll/Xbal respectively and cloned into pBluescript SKII+ and pGALL-(HIS3). Carboxyl terminally FLAG-tagged p35 and p49 were generated by PCR. FLAG tagged p35 was amplified (oligonucleotides 20 and 21) and ligated into pGALL-(HIS3) to give pGALL-(HIS3)-p35F. The intact Gal1/10 promoter was then replaced with a shorter, weaker version by inserting an Apal-BamHI fragment of pGALS-(LEU2) ${ }^{26}$ into Apal-BamHI digested pGALL-(HIS3)-p35F. Untagged p35 expressed under the control of the crippled GAL1/10 promoter was generated by inserting the Spel-Sphl fragment of pGALL-(HIS3)-p35 into pGALS(HIS3) - p35F. FLAG-tagged p49 was amplified with (oligonucleotides 22 and 23) and ligated into pGALS - (HIS3) to give pGAL-(HIS3)p49F. For expression of tagged cleavage site mutants in yeast, pGALS - (HIS3) - p35FD87A and pGALL-(HIS3)-p49F ${ }^{\text {D94 }}$ were constructed. A BamHI-Sall fragment from pGALL-(HIS3)-p35 $5^{\mathrm{D} 8 \mathrm{~A}}$ was ligated into BamHI-Sall-digested pGALS - (HIS3) - p35F to give pGALS - (HIS3)-p35F ${ }^{\mathrm{D} 87 \mathrm{~A}}$. Similarly, pGALL-(HIS3)-p49F $\mathrm{F}^{\mathrm{D} 94 \mathrm{~A}}$ was made by cloning a BamHI-Xhol fragment from pGALL(HIS3)-p49 ${ }^{\mathrm{D} 94 \mathrm{~A}}$ into BamHI-Sall-digested pGALL-(HIS3)-p49F. All amplified fragments were sequenced to verify the absence of unintentional mutations.

The sequences of the oligonucleotides referred to above were as follows:

1: GGAAGATCTACTAGTATGGCCGCTGACAGGGGACGC

2: GCTCTAGACTATGTGGGAGGGTGCCTTGGG

3: CGGGATCCATGGCAGAAGGCAACCACAGAAAAAAGCC;

4: GCTCTAGACTAATTGCCAGGAAAGAGGTAGAAATATC;

5: CGGGATCCATGTTGGAATACCTGGGCAAAGATG;

6: GCTCTAGACTAATTGCCAGGAAAGAGGTAGAAATCTC;

7: GCGGATCCATGGACTTCAGCAGAAATCTTTATGATATTGGGG;

8: GCTCTAGACTAATCAGAAGGGAAGACAAGTTTTTTTC;

9: GCGGATCCAGAATTCATGTGTGTACTGATACCAACATTC;

10: GCTCTAGAGCGGCCGCTTATATATCTATGTAAATGTTACG;

11: GCGGATCCGCCATGIGTGTACTGATACCAACATTC;

12: GCGGATCCGCCATGTGTGTAATTTTTCCGGTAG;

13: CGCGCTCGAGTTGATCAAATTGTTC;

14: GATCAACTCGAGCGCGATTACAGCGATCAAATGGCTGGATTCC;

15: GCTCTAGAGCGGCCGCTTATTTAATTGTGTTTAATATTAC;

16: CCACTTTAACTAATACTTTCAACATTTTCGG:

17: CGGCTCCACCGCCCGCGGTCACGGTCGC;

18: GCGGATCCGCGACCGTGACCGCGGGCGGTGGAGCCG;

19: CTTTATTATTTTTTATTTTATTGAGAGGGTGG;

20: GCGGATCCGCCATGTGTGTAATTTTTCCGGTAG;

21: GCTCTAGATTACTTGTCATCGTCGTCCTTGTAGTCCATTTTAATTGTGTTTAATATTACATTTTTGTTG;

22: GCGGATCCGCCATGTGTGTACTGATACCAACATTC;

23: GCTCTAGATTACTTGTCATCGTCGTCCTTGTAGTCCATTATATCTATGTAAATGTTACGATTCGCGGC;

\section{Cell lines and culture}

The glioblastoma cells lines D270, D645 and the neuro-teratocarcinoma cell line NT2 were maintained in DMEM (Life Technologies) with
$10 \%$ fetal bovine serum (FBS). D270 and D645 cells were transfected with Lipofectamine 2000 (Life Technologies) and NT2 cells with Effectene (QIAGEN) as per manufacturers' instructions.

\section{Apoptosis assays}

D270 and D645 cells were seeded and transfected as previously described. ${ }^{29}$ Briefly, $10^{5}$ cells/well were seeded in 24-well plates and co-transfected using $0.1 \mu \mathrm{g}$ of $\mathrm{CMV}-\mathrm{LacZ}$ reporter plasmid and $0.9 \mu \mathrm{g}$ of either $\mathrm{pEF}, \mathrm{pEF}-\mathrm{p} 35$ or $\mathrm{pEF}-\mathrm{p} 49$. Cells were treated with either normal media, or indicated doses of FasL, TRAIL or cisplatin. ${ }^{29}$ Cells were fixed and stained with 5-Bromo-4-chloro-3-indolyl $\beta$ - $\Delta$ galactopyranoside and blue cells were scored using established morphological apoptotic criteria. ${ }^{46}$ NT2 cells were similarly cotransfected with pEGFPN1 (Clontech) and either control or p49 expression plasmids and treated with specified doses of UV radiation. The cells were then harvested, stained with annexin- $V$ and the percentage of apoptotic, GFP positive (transfected) cells was determined using flow cytometry as previously described. ${ }^{29}$

\section{Yeast transformation and death assays}

Sacchromyces cerevisae yeast strain W303 $\alpha$ was transformed and analyzed in survival assays as previously described. ${ }^{44}$ In an attempt to produce approximately comparable lethality, copy number and promoter strength of the expression plasmids for the various caspases were manipulated. The following caspase expression plasmids were used in the yeast death assays: Caspase-2, pGALL-(LEU2)-Casp2 and PGALL-(URA)-Casp2; Caspase-3, Caspase-3-LacZ; Caspase-4, pGALL-(LEU2)-Casp4 and pGALL-(URA)-Casp4; Caspase-5, pGALL-(LEU2)-Casp5; Caspase-7, pGALL-(LEU2) Casp $7^{53}$ and pGALL-(URA)-Casp7; ${ }^{53}$ Caspase-8, pGALL(LEU2)-Casp8; DRONC, pGALS-(LEU2)-DRONC and pGALL(URA)-DRONC; drICE, pGALL-(LEU2)-rev-drICE; DCP-1, pGALL-(LEU2) - DCP-1.

For yeast-based death assays, single transformants bearing each desired combination of expression plasmids were grown until stationary phase in selective repressing medium. After washing three times in Tris $\mathrm{HCl} 10 \mathrm{mM} \mathrm{pH8}$, EDTA $1 \mathrm{mM}$ (TE), five microlitres of serial fivefold dilutions of each transformant suspension (from $\mathrm{OD}_{550}=0.086$ ) were spotted onto inducing and repressing selective minimal medim plates.

\section{Protein assays from yeast}

Transformants were grown and expression induced as previously described. ${ }^{19}$ Immunoblotting was performed as previously reported. ${ }^{19}$ For fluorometric analysis of caspase activity in yeast lysates, yeast induced for $6 \mathrm{~h}$ were pelleted, washed once in TE and resuspended in $300 \mu \mathrm{l}$ of cold lysis buffer (Tris $\mathrm{HCl} 50 \mathrm{mM} \mathrm{pH} \mathrm{7.5,} \mathrm{NaCl} 375 \mathrm{mM}$, Triton X100 1\%, EDTA $1 \mathrm{mM}$ ). After sonicating for $10 \mathrm{~s}$, the intact yeast were removed by centrifuging at $15 \mathrm{k} \mathrm{r.p.m}$. for $1 \mathrm{~min}$. The protein concentration of the supernatant was measured using the Bicinchoninic acid protein assay kit (Sigma) and samples diluted to $0.5 \mathrm{mg} / \mathrm{ml}$. Fifty microlitres of each lysate was mixed with Ac-DEVDAFC or Ac-LEHD-AFC $(50 \mu \mathrm{M})$ in activity buffer ${ }^{29}$ and the fluorescence (excitation $390 \mathrm{~nm}$, emission $520 \mathrm{~nm}$ ) measured every $2 \mathrm{~min}$ for $90 \mathrm{~min}$ and the background fluorescence was subtracted. The maximal slope of the curve was determined and the change in concentration of free AFC per minute calculated as a measure of caspase activity. 


\section{${ }^{35} \mathrm{~S}$ protein cleavage assays}

${ }^{35}$ S-labeled substrates were generated using coupled in vitro transcription/translation (TNT, Promega) and incubated with various concentrations of caspases for $1 \mathrm{~h}$ at $37^{\circ} \mathrm{C}$. Recombinant caspases were purchased from BioMol and the manufacturer's recommended reaction buffer was used. Cleavage products were resolved using $10 \%$ SDS-PAGE, transferred to Hybond-P membrane (Amersham) and the membranes subjected to autoradiography (using Kodak MR1 film).

\section{Acknowledgements}

The authors thank Dr. Nor Chejanovsky for the generous gift of the pBSKp49 plasmid. This work was funded by the Australian Research Council, National Health and Medical Research Council and James S McDonnell Foundation.

\section{References}

1. Roulston A, Marcellus R and Branton P (1999) Viruses and apoptosis. Ann. Rev. Microbiol. 53: 577-628

2. Teodoro JG and Branton PE (1997) Regulation of apoptosis by viral gene products. J. Virol. 71: 1739-1746

3. Zhou Q, Snipas S, Orth K, Muzio M, Dixit VM and Salvesen GS (1997) Target protease specificity of the viral serpin $\mathrm{CrmA}$ - analysis of five caspases. J. Biol. Chem. 272: 7797-7800

4. Deveraux $Q$ and Reed J (1999) IAP family proteins-suppressors of apoptosis. Genes Dev. 13: 239-252

5. Clem RJ, Fechheimer M and Miller LK (1991) Prevention of apoptosis by a baculovirus gene during infection of insect cells. Science 254: 1388-1390

6. Clem RJ (2001) Baculoviruses and apoptosis: the good, the bad, and the ugly. Cell Death Differ. 8: 137-143

7. Kamita SG, Majima K and Maeda S (1993) Identification and characterization of the p35 gene of Bombyx mori nuclear polyhedrosis virus that prevents virusinduced apoptosis. J. Virol. 67: 455-463

8. Morishima N, Okano K, Shibata T and Maeda S (1998) Homologous p35 proteins of baculoviruses show distinctive anti-apoptotic activities which correlate with the apoptosis-inducing activity of each virus. FEBS Lett. 427: 144-148

9. Miller L (1997) Baculovirus interaction with host apoptotic pathways. J. Cell. Physiol. 173: 178-182

10. Xue D and Horvitz HR (1995) Inhibition of the Caenorhabditis elegans cell-death protease ced-3 by a ced-3 cleavage site in baculovirus p35 protein. Nature 377 : $248-251$

11. Bump NJ, Hackett M, Hugunin M, Seshagiri S, Brady K, Chen P, Ferenz C, Franklin S, Ghayur T, LiP, Licari P, Mankovich J, Shi LF, Greenberg AH, Miller LK and Wong WW (1995) Inhibition of ICE family proteases by baculovirus antiapoptotic protein p35. Science 269: 1885-1888

12. Snipas SJ, Stennicke HR, Riedl S, Potempa J, Travis J, Barrett AJ and Salvesen GS (2001) Inhibition of distant caspase homologues by natural caspase inhibitors. Biochem. J. 357: 575-580

13. Bertin J, Mendrysa SM, Lacount DJ, Gaur S, Krebs JF, Armstrong RC, Tomaselli KJ and Friesen PD (1996) Apoptotic suppression by baculovirus p35 involves cleavage by and inhibition of a virus-induced ced-3/ICE-like protease. J Virol. 70: $6251-6259$

14. Fisher AJ, Cruz W, Zoog SJ, Schneider CL and Friesen PD (1999) Crystal structure of baculovirus p35: role of a novel reactive site loop in apoptotic caspase inhibition. EMBO J. 18: 2031-2039

15. Riedl SJ, Renatus M, Snipas SJ and Salvesen GS (2001) Mechanism-Based Inactivation of Caspases by the Apoptotic Suppressor p35. Biochemistry 40: $13274-13280$

16. dela Cruz WP, Friesen PD and Fisher AJ (2001) Crystal structure of baculovirus p35 reveals a novel conformational change in the reactive site loop after caspase cleavage. J. Biol. Chem. 276: 32933-32939
17. Xu G, Cirilli M, Huang Y, Rich RL, Myszka DG and Wu H (2001) Covalent inhibition revealed by the crystal structure of the caspase-8/p35 complex. Nature 410: $494-497$

18. Zoog SJ, Bertin J and Friesen PD (1999) Caspase inhibition by baculovirus p35 requires interaction between the reactive site loop and the beta-sheet core. J. Biol. Chem. 274: 25995-26002

19. Hawkins CJ, Yoo SJ, Petersen EP, Wang SL, Vernooy SY and Hay BA (2000) The Drosophilacaspase DRONC cleaves following glutamate or aspartate and is regulated by DIAP1, HID, and GRIM. J. Biol. Chem. 275: 27084-27093

20. Meier P, Silke J, Leevers SJ and Evan GI (2000) The Drosophila caspase DRONC is regulated by DIAP1. EMBO J. 19: 598-611

21. LaCountDJ, Hanson SF, Schneider CL and Friesen PD (2000) Caspase inhibitor p35 and inhibitor of apoptosis Op-IAP block in vivo proteolytic activation of an effector caspase at different steps. J. Biol. Chem. 275: 15657-15664

22. Vier J, Furmann $\mathrm{C}$ and Hacker G (2000) Baculovirus p35 protein does not inhibit caspase- 9 in a cell-free system of apoptosis. Biochem. Biophys. Res. Commun. 276: $855-861$

23. Ryan CA, Stennicke HR, Nava, VE, Burch, JB, Hardwick, JM and Salvesen GS (2002) Inhibitor specificity of recombinant and endogenous caspase 9. Biochem J. 366: 595-601

24. Du Q, Lehavi D, Faktor O, Qi Y and Chejanovsky N (1999) Isolation of an apoptosis suppressor gene of the Spodoptera littoralis nucleopolyhedrovirus. J. Virol. 73: 1278-1285

25. Manji GA and Friesen PD (2001) Apoptosis in motion. An apical, p35-insensitive caspase mediates programmed cell death in insect cells. J. Biol. Chem. 276: $16704-16710$

26. Hawkins CJ, Wang SL and Hay BA (1999) A cloning method to identify caspases and their regulators in yeast: identification of Drosophila IAP1 as an inhibitor of the Drosophila caspase DCP-1. Proc. Natl. Acad. Sci. USA 96: 2885-2890

27. Deveraux Q, Takahashi R, Salvesen G and Reed J (1997) X-linked IAP is a direct inhibitor of cell-death proteases. Nature 388: 300-304

28. Hawkins CJ, Silke J, Verhagen AM, Foster R, Ekert PG and Ashley DM (2001) Analysis of candidate antagonists of IAP-mediated caspase inhibition using yeast reconstituted with the mammalian Apaf-1-activated apoptosis mechanism Apoptosis 6: $331-338$

29. KnightMJ, Riffkin CD, Muscat AM, Ashley DM and Hawkins CJ(2001) Analysis of FasL and TRAIL induced apoptosis pathways in glioma cells. Oncogene 20 5789-5798

30. Silke J, Ekert PG, Day CL, Hawkins CJ, Baca M, Chew J, Pakusch M, Verhagen AM and Vaux DL (2001) Direct inhibition of caspase 3 is dispensable for the antiapoptotic activity of XIAP. EMBO J. 20: $3114-3123$

31. Dorstyn L, Colussi P, Quinn L, Richardson H and Kumar S (1999) DRONC, an ecdysone-inducible Drosophila caspase. Proc. Natl. Acad. Sci. USA 96: 4307 4312

32. Hay BA, Wolff T and Rubin GM (1994) Expression of baculovirus p35 prevents cell death in Drosophila. Development 120: 2121-2129

33. Sugimoto A, Friesen PD and Rothman JH (1994) Baculovirus p35 prevents developmentally programmed cell death and rescues a ced-9 mutant in the nematode Caenorhabditis elegans. EMBO J. 13: 2023-2028

34. Rabizadeh S, Lacount DJ, Friesen PD and Bredesen DE (1993) Expression of the baculovirus p35 gene inhibits mammalian neural cell death. J. Neurochem. 61: $2318-2321$

35. Beidler DR, Tewari M, Friesen PD, Poirier G and Dixit VM (1995) The baculovirus p35 protein inhibits Fas- and Tumor necrosis factor-induced apoptosis. J. Biol. Chem. 270: 16526-16528

36. Martinou I, Fernandez PA, Missotten M, White E, Allet B, Sadoul R and Martinou JC (1995) Viral proteins E1B19k and p35 protect sympathetic neurons from cell death induced by NGF deprivation. J. Cell. Biol. 128: 201-208

37. Robertson NM, Zangrilli J, Fernandes-Alnemri T, Friesen PD, Litwack G and AlnemriES (1997) Baculovirus p35 inhibits the glucocorticoid-mediated pathway of cell death. Cancer Res. 57: 43-47

38. Izquierdo M, Grandien A, Criado LM, Robles S, Leonardo E, Albar JP, de Buitrago GG and Martinez AC (1999) Blocked negative selection of developing T cells in mice expressing the baculovirus p35 caspase inhibitor. EMBO J. 18: $156-166$ 
39. Shibata M, Hisahara S, Hara H, Yamawaki T, Fukuuchi Y, Yuan J, Okano H and Miura M (2000) Caspases determine the vulnerability of oligodendrocytes in the ischemic brain. J. Clin. Invest. 106: 643-653

40. Araki T, Shibata M, Takano R, Hisahara S, Imamura S, Fukuuchi Y, Saruta T, Okano Hand Miura M (2000)Conditional expression of anti-apoptotic protein p35 by Cre-mediated DNA recombination in cardiomyocytes from loxP-p35transgenic mice. Cell Death Differ. 7: 485-492

41. Hisahara S, Araki T, Sugiyama F, Yagami K, Suzuki M, Abe K, Yamamura K, Miyazaki J, Momoi T, Saruta T, Bernard CC, Okano H and Miura M (2000) Targeted expression of baculovirus p35 caspase inhibitor in oligodendrocytes protects mice against autoimmune-mediated demyelination. EMBO J. 19:341 348

42. Viswanath V, Wu Z, Fonck C, Wei Q, Boonplueang R and Andersen JK (2000) Transgenic mice neuronally expressing baculoviral p35 are resistant to diverse types of induced apoptosis, including seizure-associated neurodegeneration. Proc. Natl. Acad. Sci. USA 97: 2270-2275
43. Doerfler P, Forbush KA and Perlmutter RM (2000) Caspase enzyme activity is not essential for apoptosis during thymocyte development. J. Immunol. 164: 4071-4079

44. Hawkins CJ, Wang SL and Hay BA (2000) Monitoring activity of caspases and their regulators in yeast Saccharomyces cerevisiae. Methods Enzymol. 322: $162-174$

45. Hawkins CJ, Uren AG, Hacker G, Medcalf RL and Vaux DL (1996) Inhibition of interleukin 1-beta-converting enzyme-mediated apoptosis of mammalian cells by baculovirus IAP. Proc. Natl. Acad. Sci. USA 93: 13786-13790

46. Miura M, Zhu H, Rotello R, Hartweig EA and Yuan J (1993) Induction of apoptosis in fibroblasts by IL-1 $\beta$-converting enzyme, a mammalian homolog of the $C$. elegans cell death gene ced-3. Cell 75: 653-660 